\title{
Atomic environment and interfacial structural order of TiAlN/Mo multilayers
}

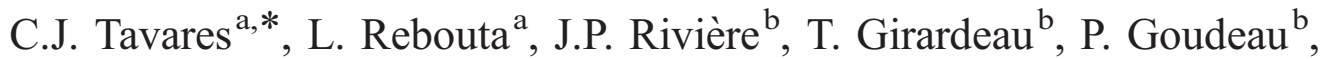 \\ E. Alves ${ }^{\text {c }}$, N.P. Barradas ${ }^{\mathrm{c}}$ \\ ${ }^{a}$ Departamento de Física (GRF), Universidade do Minho, Azurém, 4800-058 Guimarães, Portugal \\ ${ }^{\mathrm{b}}$ Lab. de Metallurgie Physique, Université de Poitiers, 86962 Futuroscope, France \\ 'Instituto Tecnológico e Nuclear, EN 10, 2686-953 Sacavém, Portugal
}

Received 3 October 2003; accepted 26 March 2004

Available online 28 July 2004

\begin{abstract}
Multilayered TiAlN/Mo coatings were deposited by dc reactive magnetron sputtering in a custom-made chamber. In order to assess the composition of these coatings, a combined study of Extended X-ray Absorption Fine Structure (EXAFS) and Rutherford Backscattering Spectrometry (RBS) experiments were performed. Through the simulation of the EXAFS spectra, giving the local environment of the titanium atoms inside the nitride (TiAlN), a cubic phase has been evidenced with aluminium atoms occupying titanium sites. For modulation periods in the range of 3.6-11.8 nm, RBS simulations on these multilayers also enabled the determination of the level of intermixing that occurs at the interfaces as a function of the negative bias voltage and number of layers. It was observed that the intermixing width could be as high as $2.1 \mathrm{~nm}$ for the roughest samples (larger periods) or as low as $0.4 \mathrm{~nm}$ for those with the sharpest interfaces (smaller periods).
\end{abstract}

(C) 2004 Elsevier B.V. All rights reserved.

Keywords: Multilayers; EXAFS; RBS; Simulations; Roughness; Atomic environment

\section{Introduction}

Multilayer coating design is a field of never-ending solutions for a vast range of applications [1]. In order to optimise the mechanical performance of these structures for the wear protection coating industry, the mechanical and physical properties need to be understood. In our case, we combine alternate layers of a hard nitride (TiAlN) together with a softer metal (Mo). When using a ductile layer in the bilayer system, this stacking feature should improve the strength of the coating by inhibiting crack propagation through the introduction of multiple interfaces capable of deflecting or absorbing the crack tips.

The investigation for the optimum deposition parameters undergoes inevitable microstructural changes in the alloy formation of TiAlN that can be either extended or at an atomic scale. Despite X-ray diffraction (XRD) being a very versatile structural probing tool, it is however not well-

\footnotetext{
* Corresponding author. Tel.: +351-253-510474; fax: +351-253510461.

E-mail address: ctavares@fisica.uminho.pt (C.J. Tavares).
}

suited for analysing the atomic environment. The Extended $\mathrm{X}$-ray Absorption Fine Structure (EXAFS) is a well-known technique able to finely characterize the local order around the absorbing atom. This effect is detected as an oscillatory structure in the X-ray absorption coefficient (on a large energy scale), and these oscillations contain quantitative information about the local environment of the absorbing atom. Subsequent modelling of the EXAFS spectra yields valuable information on the chemical bonds and the average interatomic distances from the central atom to the nearest neighbours.

On the other hand, Rutherford Backscattering Spectrometry (RBS) has also been used to study both the composition of the TiAlN/Mo multilayers and the average interlayer roughness as a function of the number of bilayers and of the multilayer period $(\Lambda)$ [2-4]. Using grazing angles for both ingoing and outgoing ions in the RBS analysis, it was possible to increase the depth resolution and hence the sensitivity to roughness in this technique.

In this study we combine both EXAFS and RBS in grazing geometry to profile the interface roughness and mixing of TiAlN/Mo multilayers at an atomic level. RBS 
Table 1

Important deposition parameters for the production of the PVD-grown TiAlN/Mo multilayers

\begin{tabular}{ll}
\hline Base pressure & $5 \times 10^{-5} \mathrm{~Pa}$ \\
Argon partial pressure (TiAlN) & $0.35 \mathrm{~Pa}$ \\
Argon partial pressure (Mo) & $0.5 \mathrm{~Pa}$ \\
Nitrogen partial pressure (TiAlN) & $0.1 \mathrm{~Pa}$ \\
Temperature during deposition & $250{ }^{\circ} \mathrm{C}$ \\
Target-to-substrate distance & $65 \mathrm{~mm}$ \\
Bias voltage range & 0 to $-120 \mathrm{~V}$ \\
Modulation period range & $3.6-11.8 \mathrm{~nm}$ \\
Relative thickness of the Mo layer in a bilayer & $\sim 56 \%$ \\
\hline
\end{tabular}

simulations were performed with a code where roughness is explicitly taken into account [5]. Using Mo and $\mathrm{Ti}_{0.4} \mathrm{Al}_{0.6} \mathrm{~N}$ densities, the modulation period and individual layer thickness were also evaluated.

\section{Experimental details}

TiAlN/Mo multilayer coatings were deposited on (100) silicon wafers using a custom-made reactive sputtering automated system operating in static mode, with the experimental conditions presented in Table 1. An automated gas inlet system enabled the continuous adjustment of the $\mathrm{Ar} / \mathrm{N}_{2}$ atmosphere, nitrogen being introduced only during the nitride deposition. Before deposition, the substrates were in situ sputter-etched in an argon atmosphere. Additional information on these experimental techniques can be found elsewhere [6,7].

RBS experiments were performed using a $2.0 \mathrm{MeV} \mathrm{He}$ beam from a 2.5 MV Van de Graaff accelerator. The backscattered particles were detected by a surface barrier detector with an energy resolution of $15 \mathrm{keV}$ at FWHM placed at $160^{\circ}$ to the beam direction, in the Cornell geometry. The beam spot was $0.2 \times 0.6 \mathrm{~mm}^{2}$. The energy resolution at the surface was $1 \mathrm{~nm}$ for $85^{\circ}$ grazing angle of incidence, as calculated with the code DEPTH [8]. The IBA DataFurnace code $[9,10]$ was used to simulate the RBS spectra. Roughness leads to a broadening of spectral features. Quantitative determination of roughness can be made by comparing the width of the multilayer peaks to the width they would have if no layer roughness or intermixing would be present. The implementation of roughness models in the simulations is described in detail elsewhere [5]. The main assumptions used in this work are that all interfaces are equivalent, and that the roughness or mixing follows a Gaussian distribution.

$\mathrm{X}$-ray absorption measurements were performed at LURE (Orsay, France) using the synchrotron radiation from the DCI storage ring operating with an energy of $1.85 \mathrm{GeV}$ and a current of $230 \mathrm{~mA}$. The absorption spectra were collected over $1000 \mathrm{eV}$ above Ti-K edge $(4970 \mathrm{eV})$ on EXAFS-I station (D-42 beam line) equipped with a Si(111) double crystal monochromator. The conversion electron mode was used since the thickness of the studied films is of the order of the probe depth CEEXAFS technique (typically $70 \mathrm{~nm}$ ). The electron yield spectra were recorded by using a detector operating at liquid nitrogen temperature [11]. This device allowed EXAFS amplitude improvements by reduction of thermal agitation. The EXAFS oscillations were analysed according to a standard procedure described in detail in Refs. [12,13]. Fourier transformation was performed over the range $2-12 \AA^{-1}$ using nodal points for the Hamming window function, $k^{3}$ weighted. The EXAFS contributions of the different coordination shells can be separated, singled out and back-transformed in $k$ space; then their real part was simulated using formula (1), reported later.

\section{Results and discussion}

The EXAFS technique consists in a measurement of the $\mathrm{X}$-ray absorption coefficient on an energy range including the edge energy of the chosen chemical specie. For illustration, the raw data of a $(\mathrm{TiAlN}) / \mathrm{Mo} \times 100$ multilayer measured at Ti-K edge are given in Fig. 1 (for the sake of clarity, these data are normalised to 1 above the absorption edge). The principle of EXAFS relies on the behaviour of the electron ejected from a central atom after an X-ray photon has been absorbed. The outgoing wave function of the ejected electron is then backscattered by the surrounding atoms giving rise to a system of constructive or destructive interferences depending on the wavelength of the incident $\mathrm{X}$-ray beam and the distances to the neighbouring atoms. In the most recent theories, the EXAFS signal is treated in terms of curved waves and theoretical simulations take into account the multiple scattering of the photoelectron [14]. Nevertheless, in this work, the EXAFS phenomenon is analysed using the single scattering EXAFS formula since, when the study is limited to the first two peaks of the Fourier transform, the multiple scattering can be neglected.

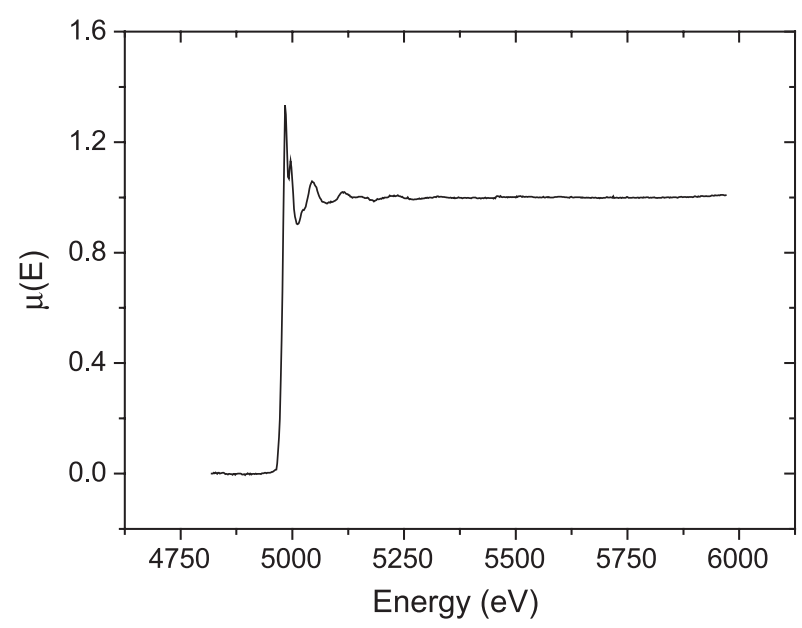

Fig. 1. Ti-K edge absorption coefficient as a function of X-ray energy measured for a $($ TiAlN/Mo $) \times 100$ multilayer sample with $\Lambda=7.9 \mathrm{~nm}$. 
The information appears as structural parameters in the plane-wave EXAFS signal $k \chi_{i}$ [15], together with other quantities which depend on the electronic structure of the material:

$$
\begin{aligned}
k \chi_{i}(k)= & -\sum_{j} \frac{N_{j}}{r_{i j}^{2}}\left|f_{j}(\pi, k)\right| \cdot \exp \left(-2 \sigma_{i j}^{2} k^{2}\right) \\
& \cdot \exp \left(-2 \Gamma \frac{r_{i j}}{k}\right) \cdot \sin \left(2 k r_{i j}+\phi_{i j}(k)\right)
\end{aligned}
$$

where $k$ is the wave number, $N_{j}\left|f_{j}(\pi, k)\right|$ is the backscattering amplitude of $N_{j}$ atoms of type $j$ surrounding the absorbing atom of species $i$, a distance $r_{i j}$ apart; $\sigma_{i j}^{2}$ is the mean square relative displacement (MSRD) assuming a Gaussian distribution; the term $\exp \left(-2 \sigma_{i j}^{2} k^{2}\right)$ is also called the Debye-Waller factor; $\exp \left(-2 \Gamma r_{i j} / k\right)$ is a mean free path term taking into account inelastic effects; $\phi_{i j}(k)$ is the sum of the central and backscattering phase shift. The amplitude $\left|f_{j}(\pi, k)\right|$ and the total phase shifts $\phi_{i j}(k)$ used here are theoretical values calculated by McKale et al. [16]. These parameters, successfully tested on $\mathrm{Ti}$, TiN and TiAl reference samples, are very similar from those extracted from the FEFF software package [14], which takes into account multiple scattering paths.

In Fig. 2, one can see the radial distribution Fourier transform extracted from the X-ray absorption spectrum measured at the Ti-K edge on a (TiAlN/Mo $\times 100$ multilayer sample. This sample was produced in the same conditions as in Table 1, with a modulation period of 7.9 $\mathrm{nm}$ and grown with a bias voltage of $-60 \mathrm{~V}$. For comparison, the TiN reference sample spectrum has also been included. The symbol $(*)$ marks the maximum corresponding to the first neighbours (first shell) of Ti, that is assigned to the nitrogen atoms. The symbol (**) marks the maximum corresponding to the second shell of nearest neighbours of $\mathrm{Ti}$, which happen to be $\mathrm{Ti}-\mathrm{Ti}$ bonds according to the reference sample.

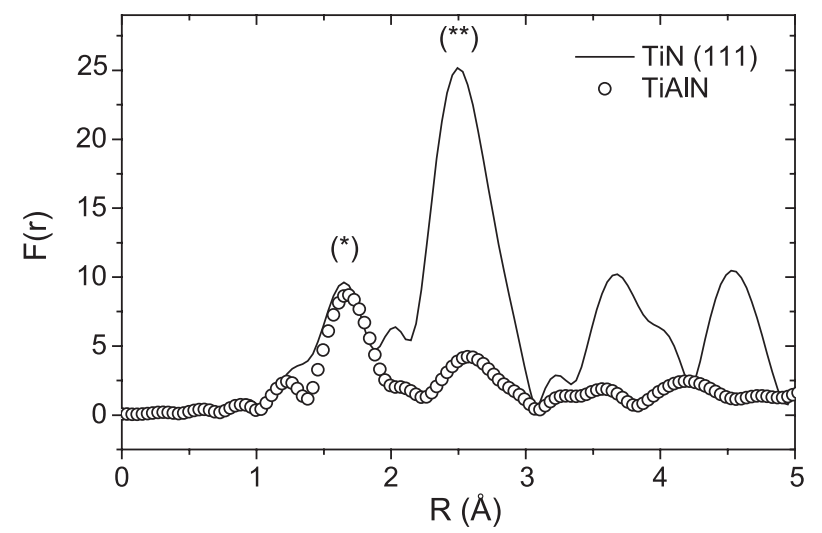

Fig. 2. Radial distribution Fourier transform at the Ti-K edge measured on a $($ TiAlN/Mo) $\times 100$ multilayer sample (with $\Lambda=7.9 \mathrm{~nm}$ ) compared with a TiN (111) reference sample. The first maximum $(*)$ is attributed to the first coordination shell, which is assigned with the $\mathrm{Ti}-\mathrm{N}$ bond, whereas the second maximum $\left({ }^{* *}\right)$ refers to the $\mathrm{Ti}-\mathrm{Ti}$ and $\mathrm{Ti}-\mathrm{Al}$ bonds.

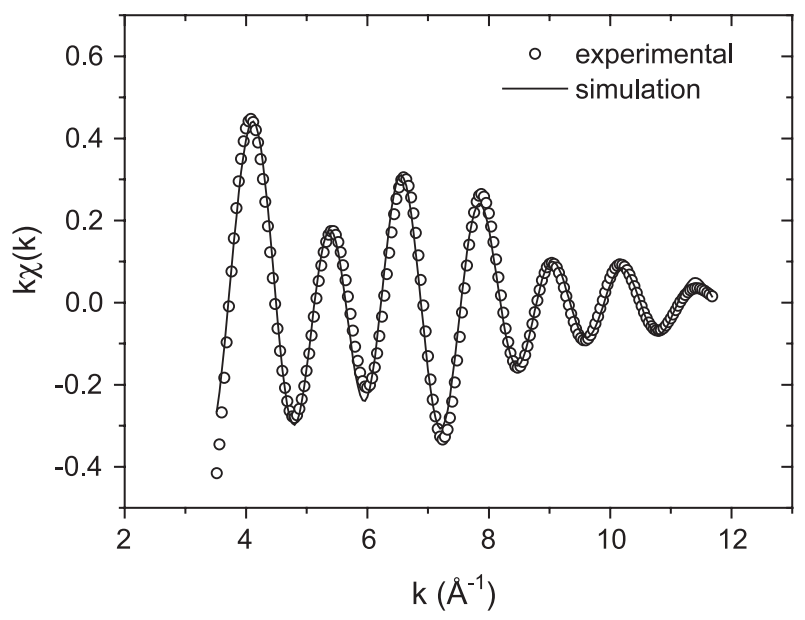

Fig. 3. Simulation of the experimental EXAFS filtered spectra of a TiN reference sample. Extracted parameters are reported in Table 2.

The simulation of the filtered experimental EXAFS spectrum (limited to peaks noted $*$ and ${ }^{* *}$ ) of the TiN reference sample is shown in Fig. 3. The structural parameters related to this fit, an estimation of its uncertainties, the amplitude reduction factor $\left(S_{0}^{2}\right)$, shift energy $\left(E_{0}\right)$ and the residue value (which characterize the quality of the simulation) are given in Table 2. One can notice that the expected environment $(6 \mathrm{~N}$ atoms located at $2.14 \AA, 12 \mathrm{Ti}$ atoms located at $3.0 \AA$ ) is obtained. The low value of the residue confirms that the retro-diffusion parameters (amplitude and phase shift) that were used are suitable. It also indicates the validity of a single scattering treatment on this kind of structure, even on the second coordination shell. The first peak of the studied (TiAlN / Mo) $\times 100$ sample, characteristic of the first Ti-N coordination shell, is clearly the same as on the reference sample (Fig. 2). The agreement between the experimental signal characteristic of this peak and the theoretical one (Fig. 4), obtained with six atoms of nitrogen located at $2.15 \AA$ from the central atom, confirms that the Ti atoms are completely surrounded by $\mathrm{N}$ atoms. Consequently, Al atoms substitute $\mathrm{Ti}$ in the TiAlN phase. All the parameters extracted from this previous simulation are reported in Table 2. Additionally, the existence of a hexagonal AlN phase with Ti substitutional atoms on Al lattice can be ruled out because in that case the Ti-N bond length should be $1.9 \AA$; as it was confirmed through the XRD patterns of the same sample [6,7].

The important attenuation of the second peak amplitude in the case of the studied sample (see Fig. 2, peak noted ${ }^{* *}$ ) confirms the existence of $\mathrm{Al}$ substitutional atoms on a $\mathrm{Ti}$ lattice. Indeed, the $\mathrm{Ti}-\mathrm{Ti}$ and $\mathrm{Ti}-\mathrm{Al}$ phase shifts $\phi_{i j}(k)$ that appear in the EXAFS formulation are out-of-phase, thus leading to a destructive interference and subsequent decrease of the EXAFS amplitude. In order to confirm this qualitative study, a simulation of the EXAFS and Fourier transform spectra of the second peak has been performed taking into account the composition derived from the RBS experiments: $\mathrm{Ti}_{0.4} \mathrm{Al}_{0.6} \mathrm{~N}$. For the second shell, corresponding to 12 atoms 
Table 2

EXAFS simulation results on a TiN reference sample and on a (TiAlN/Mo) $\times 100$ multilayer sample with a modulation period of 7.9 nm and grown with a bias voltage of $-60 \mathrm{~V}$

\begin{tabular}{|c|c|c|c|c|c|}
\hline & $\begin{array}{c}\text { Chemical } \\
\text { specie }\end{array}$ & $\begin{array}{l}\text { Number of } \\
\text { neighbours }\end{array}$ & Distance $(\AA)$ & $\operatorname{MSRD}(\AA)$ & $\begin{array}{c}E_{0}(\mathrm{eV}) \\
S_{0}^{2} \\
\text { Residue }\end{array}$ \\
\hline TiN reference sample & $\begin{array}{l}\mathrm{N} \\
\mathrm{Ti}\end{array}$ & $\begin{array}{r}6 \pm 2 \\
12 \pm 1\end{array}$ & $\begin{array}{r}2.14 \pm 0.05 \\
3.0 \pm 0.02\end{array}$ & $\begin{array}{c}.07 \pm 0.01 \\
.075 \pm 0.005\end{array}$ & $\begin{array}{c}3 \\
1 \\
0.02\end{array}$ \\
\hline (TiAlN)/Mo sample & $\mathrm{N}$ & $6 \pm 1$ & $2.15 \pm 0.05$ & $.075 \pm 0.01$ & $\begin{array}{c}2 \\
1 \\
0.3\end{array}$ \\
\hline & $\begin{array}{l}\mathrm{Ti} \\
\mathrm{Al}\end{array}$ & $\begin{array}{l}4.8 \\
7.2\end{array}$ & $\begin{array}{l}2.93 \pm .03 \\
2.94 \pm .02\end{array}$ & $\begin{array}{r}.08 \pm 0.005 \\
.086 \pm 0.005\end{array}$ & $\begin{array}{c}5 \\
1 \\
0.07\end{array}$ \\
\hline
\end{tabular}

for a fcc lattice, this composition leads to 4.8 titanium atoms and 7.2 aluminium atoms. The quality of the simulation shown in Fig. 5 with these coordination numbers validates the composition extracted from the RBS measurements. The other parameters related to this simulation are reported on Table 2. The rather low values of the MSRD, and close to the ones obtained for the TiN reference sample, are characteristic of a well crystallized phase. The lattice parameter $(a=4.14 \AA)$ extracted from the next nearest neighbours distance of the TiAlN phase $(2.93 \AA)$ is fairly in agreement with the one obtained through the XRD patterns of the same sample [6,7]. From these different results, one can conclude that TiAlN has a $\mathrm{NaCl} \mathrm{B} 1$-type TiN phase, where $\mathrm{Al}$ atoms substitute $\mathrm{Ti}$ atoms. However, the lattice parameter of the TiAlN phase is lower than the one attributed to TiN (4.24 $\AA$ ) [17]. This discrepancy can be justified since $\mathrm{Al}$ atoms are smaller than the Ti atoms. One can also add that the presence of Mo next-nearest neighbour in some $\mathrm{Ti}$ environments cannot be ruled out since it can contribute to the reduction of the EXAFS amplitude of the second peak.

The Mo layers are quite homogeneous, resulting in a composition of $\sim 99 \%$, which is indicative of very low

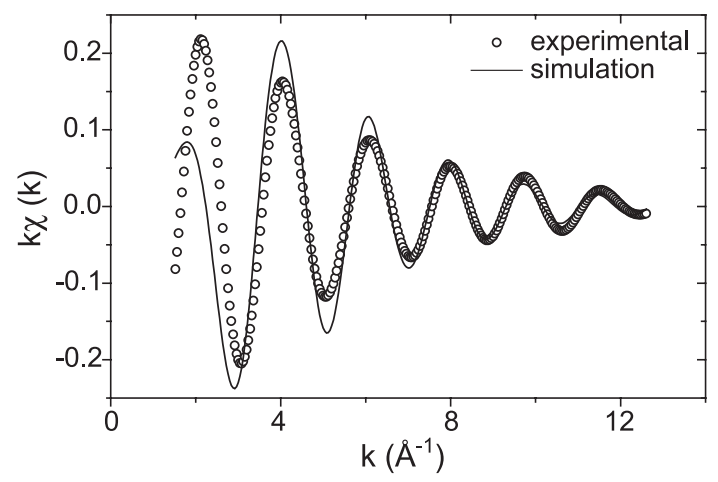

Fig. 4. Simulation of the EXAFS filtered spectra corresponding to the first neighbours (first shell) of Ti, shown in Fig. 2. Extracted parameters are reported in Table 2. oxygen content. For the determination of the individual layer thickness and calculation of the elemental percentage, in Table 3 , the densities that were used $\left(\rho_{\text {TiAIN }}=9.9 \times 10^{22}\right.$ atoms $/ \mathrm{cm}^{2}$ and $\rho_{\mathrm{Mo}}=6.4 \times 10^{22}$ atoms $/ \mathrm{cm}^{2}$ ) were obtained from the RBS fits to monolithic thin films of TiAlN and Mo grown in the same conditions. From the results in Table 3,

a

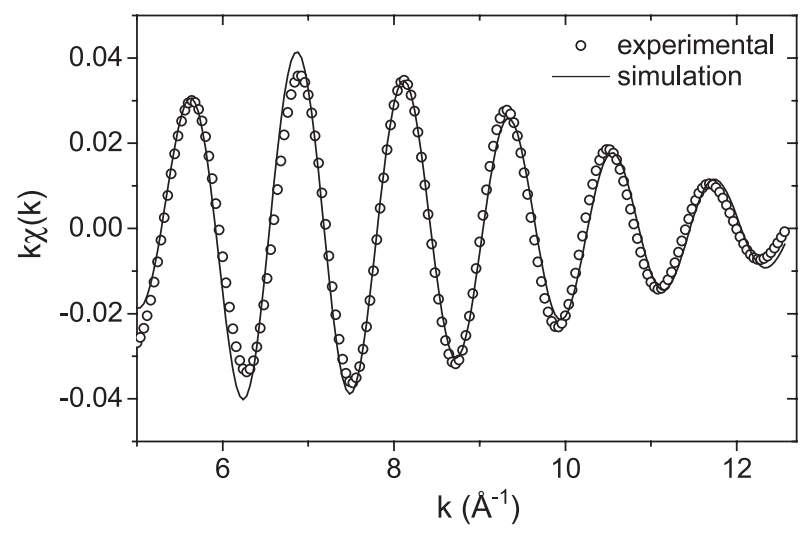

b

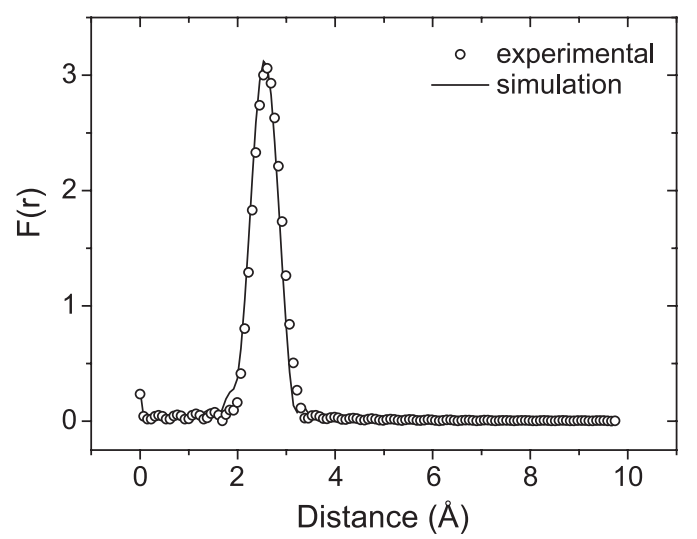

Fig. 5. Simulation of the experimental spectra of a TiAlN reference sample: (a) EXAFS filtered spectrum simulation; (b) Fourier transform simulation. Extracted parameters are reported in Table 2. 
Table 3

RBS composition simulation results from a series of $($ TiAlN/Mo $) \times 50$ multilayer samples grown with in the same conditions as stated in Table 1 , except for the bias voltage

\begin{tabular}{|c|c|c|c|c|c|c|c|c|}
\hline$\Lambda(\mathrm{nm})$ & $t\left(\mathrm{at} / \mathrm{cm}^{2}\right)$ & Mo (at.\%) & Ti (at.\%) & $\mathrm{Al}($ at. $\%)$ & N (at.\%) & $t_{\mathrm{TiAlN}} / t_{\mathrm{Mo}}(\mathrm{nm})$ & Bias (V) & Composition \\
\hline 11.8 & $4.9 \times 10^{18}$ & 37 & 13 & 19 & 31 & $6.2 / 5.6$ & 0 & $\mathrm{Ti}_{0.40} \mathrm{Al}_{0.60} \mathrm{~N}_{1.0}$ \\
\hline 11.0 & $4.4 \times 10^{18}$ & 42 & 11.5 & 17.5 & 29 & $5.2 / 5.8$ & -60 & $\mathrm{Ti}_{0.40} \mathrm{Al}_{0.60} \mathrm{~N}_{1.0}$ \\
\hline 10.5 & $4.2 \times 10^{18}$ & 36 & 13 & 19 & 32 & $5.3 / 5.2$ & -120 & $\mathrm{Ti}_{0.41} \mathrm{Al}_{0.59} \mathrm{~N}_{1.0}$ \\
\hline
\end{tabular}

The elemental compositions are in atomic percent. $\Lambda$ is the modulation periodicity, while $t_{\mathrm{TiAlN}}$, and $t_{\mathrm{Mo}}$ represent, respectively, the nitride and Mo thickness, $t$ being the atomic density of the multilayer.

one notices that there is a significant decrease in the nitride layer thickness $\left(t_{\mathrm{TiAlN}}\right)$ as the bias voltage changes from 0 to $-60 \mathrm{~V}$, which is due to the enhancement of the flux of heavier ions and of the ionic energy of $\mathrm{Ar}^{+}$. Additionally, as the negative bias is increased, a re-sputtering mechanism resulting from the increase of the ion bombardment takes place in the substrates and accounts for the drop in layer thickness.

The interfaces of these TiAlN/Mo multilayered structures depend strongly on the nucleation and growth mechanisms of each layer on the previous one, and on their physical and chemical interaction. Understanding the origin of these effects requires knowledge of the interface structure, where the interfacial roughness is of prime importance. Following this, RBS was used to study the average interlayer roughness as a function of the number of bilayers for a particular series of TiAlN/Mo multilayers grown in the same conditions, with a bias voltage of $-100 \mathrm{~V}$ and with a periodicity $\Lambda \approx 3.6 \mathrm{~nm}$. The calculated standard interfacial width $(\sigma)$ of these films has revealed that this roughness is practically unchanged with the increasing number of bilayers from $10(\sigma=0.5 \mathrm{~nm})$ to $50(\sigma=0.6 \mathrm{~nm})$, hence revealing smooth planar interfaces. In Fig. 6, the RBS spectra was recorded for the thinnest sample (10 bilayers), at different tilt angles from the surface normal. As the tilt angle increases from $75^{\circ}$ to $85^{\circ}$ the in-depth resolution also increases as a function of $1 / \cos \theta$ and therefore the modulation inherent to the first layers is discerned. The simulation (solid line) was performed in order to obtain the individual layer thickness and the modulation period. Except for the higher tilt angle $\left(85^{\circ}\right)$, the interfacial roughness was not taken into account.

In Fig. 6, for the tilt angle of $85^{\circ}$ with enhanced in-depth resolution, a simulation has been performed in order to determine the interfacial width which is indicative of the level of chemical intermixing from TiAlN and Mo at the interfaces. Since the outermost layer of the multilayer is a Mo layer, the backscattered signal from the Ti atoms in the first $\mathrm{Ti}_{0.4} \mathrm{Al}_{0.6} \mathrm{~N}$ layer is shifted towards lower energies. A RBS simulation was performed assuming a standard interface width of $\sigma=0.4 \mathrm{~nm}$, which in turn was obtained from the element depth profile mixing measured by RBS. An interface width of this order is indicative that the interface has a low content of chemical intermixing.

On the other hand, similar calculations performed on a sample with a larger periodicity $(\Lambda=11.1 \mathrm{~nm}, 50$ bilayers, bias $=-60 \mathrm{~V}$ ) resulted in larger interfacial width of $\sigma=2.1$ $\mathrm{nm}$, as seen on Fig. 7. It seems that the limiting size of the bilayer inhibits a three-dimensional growth and thus prevents the enhancement of the waviness of the interfaces. On this last sample, the roughness value was higher due to two important factors: target-to-substrate distance and bias voltage. It was produced with a target-to-substrate distance higher $(110 \mathrm{~mm})$ than the lower-period samples $(65 \mathrm{~mm})$, being this distance factor responsible for the variation of the $\mathrm{Ti}_{0.4} \mathrm{Al}_{0.6} \mathrm{~N}$ layer crystalline texture from a weak [111] to a weak [100] preferred orientation. This re-orientation of crystal structure plays an important role in the accommodation of internal stress and interfacial mismatch between the two lattices. The difference in the average lattice parameters of $\mathrm{Ti}_{0.4} \mathrm{Al}_{0.6} \mathrm{~N}$ and $\mathrm{Mo}$, measured by XRD, correspond to an optimised lattice misfit of about $8 \%$, upon a $45^{\circ}$ rotation of the crystalline cubic cells of both materials. Additionally,

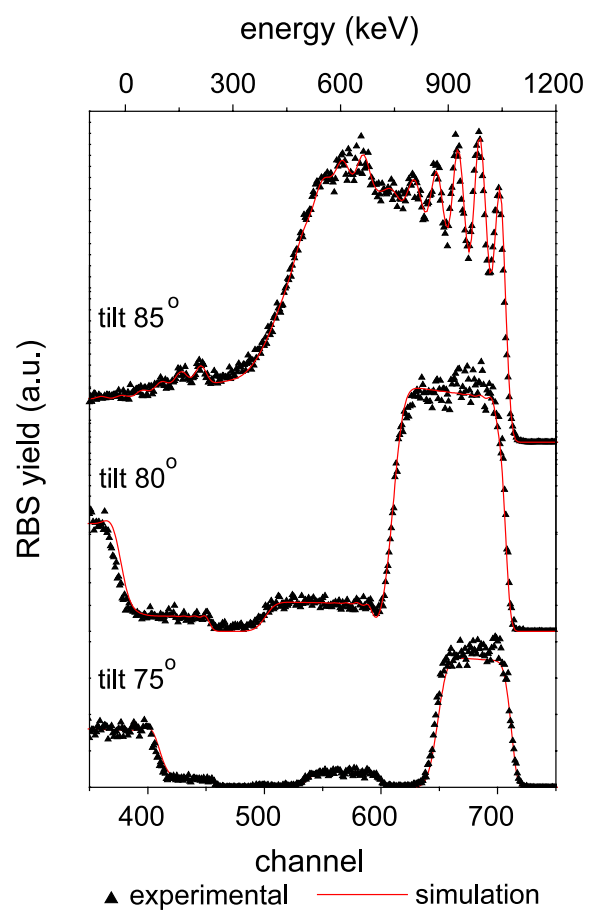

Fig. 6. RBS spectra recorded on a $\left(\mathrm{Ti}_{0.4} \mathrm{Al}_{0.6} \mathrm{~N} / \mathrm{Mo}\right) \times 10$ multilayer with a modulation period of $3.6 \mathrm{~nm}$ at different tilt angles from the surface normal. The simulation (solid line) was performed in order to obtain the individual layer thickness and the modulation period. For the case of a tilt angle of $85^{\circ}$, the simulation was performed assuming an interlayer mixing (standard interfacial width) of $0.4 \mathrm{~nm}$ at the interface. 


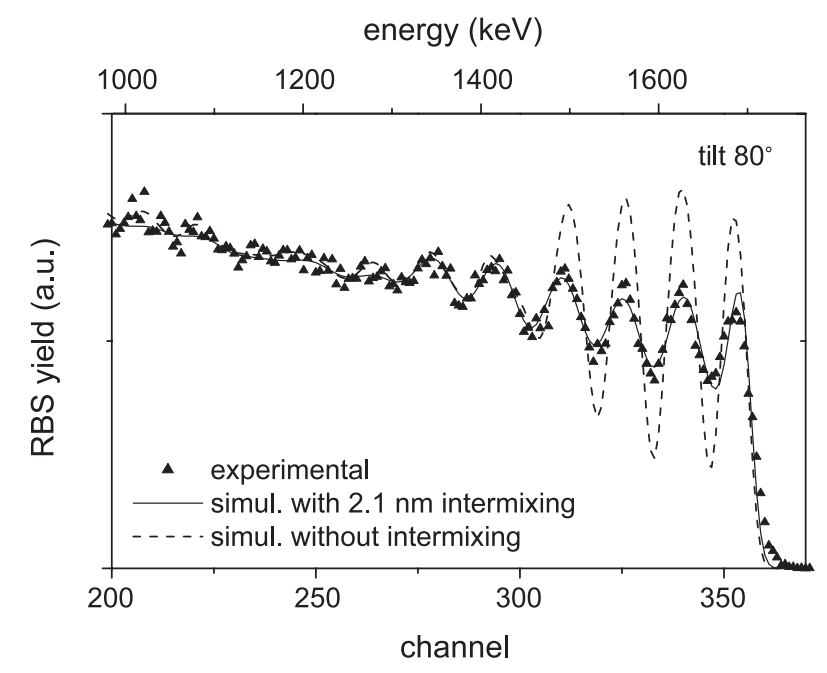

Fig. 7. RBS spectra recorded on a $\left(\mathrm{Ti}_{0.4} \mathrm{Al}_{0.6} \mathrm{~N} / \mathrm{Mo}\right) \times 50$ multilayer with a modulation period of $11.1 \mathrm{~nm}$ at a tilt angle of $80^{\circ}$. The dotted line represents the RBS simulation assuming abrupt interfaces, while the solid line is the RBS simulation assuming a mixing (standard interfacial width) of $2.1 \mathrm{~nm}$ at the interfaces.

the change in texture from (111) to (100) in this multilayer system is also achieved by increasing the negative bias potential [7], thus enhancing the ion bombardment and flattening of the surface topography; as was the case of the lower-period samples grown with a bias of $-100 \mathrm{~V}$.

\section{Conclusions}

Experimental and numerical EXAFS and RBS techniques enabled separate determination, with a good agreement, of the average composition of TiAlN/Mo multilayers. Additionally, RBS proved to be very useful as a structural tool to evaluate the level of chemical mixing at the interfaces. From the results, it was concluded that this level of intermixing decreased as the multilayers were grown with smaller periods, due to the fact that the limiting size of the bilayer inhibits a three-dimensional growth and thus prevents the enhancement of the waviness of the interfaces. The mechanism involved with surface roughening is mainly related to growth mechanisms, which are in turn related to deposition parameters, such as bias voltage, working pressure, the relatively low substrate temperature during deposition, and the target-to-substrate distance. From this, it was concluded that both a decrease in target-to-substrate distance and an increase in the negative bias potential led to the growth of sharper interfaces within the multilayer.

\section{Acknowledgements}

The authors gratefully acknowledge the financial support of the FCT institution under the project no. POCTI/32670/CTM/2000 co-financed by European community fund FEDER, and from the FCT/MCT pluri-annual program. The authors also acknowledge the financial support from French/Portuguese France Embassy/ICCTI bilateral agreement (project no. $543 \mathrm{~B} 3 / 2001)$ ). We wish to thank A. Traverse for her help in conducting the EXAFS measurements and the technical staff of LURE-DCI for providing the synchrotron beam and for assistance with the experiments.

\section{References}

[1] P.Eh. Hovsepian, W.-D. Münz, Vacuum 69 (2003) 27.

[2] N.P. Barradas, J.C. Soares, M.F. da Silva, F. Pászti, E. Szilágyi, Nucl. Instrum. Methods B 94 (1994) 266.

[3] C.J. Tavares, L. Rebouta, E.J. Alves, N.P. Barradas, J. Pacaud, J.P. Rivière, Nucl. Instrum. Methods B 188 (2002) 90.

[4] R. Behrisch, S. Grigull, U. Kreissig, R. Grötzschel, Nucl. Instrum. Methods B 136-138 (1998) 628.

[5] N.P. Barradas, J. Phys., D, Appl. Phys. 34 (2001) 2109.

[6] C.J. Tavares, L. Rebouta, E. Alves, A. Cavaleiro, P. Goudeau, J.P. Rivière, A. Declemy, Thin Solid Films 377-378 (2000) 425.

[7] C.J. Tavares, L. Rebouta, J.P. Rivière, J. Pacaud, H. Garem, K. Pischow, Z. Wang, Thin Solid Films 398-399 (2001) 397.

[8] E. Szilágyi, Nucl. Instrum. Methods Phys. Res., B Beam Interact. Mater. Atoms 161-163 (2000) 37.

[9] N.P. Barradas, C. Jeynes, R.P. Webb, Appl. Phys. Lett. 71 (1997) 291.

[10] N.P. Barradas, C. Jeynes, R.P. Webb, U. Kreissig, R. Grötzschel, Nucl. Instrum. Methods B 149 (1999) 233.

[11] J. Mimault, J.J. Faix, T. Girardeau, M. Jaouen, G. Tourillon, Meas. Sci. Technol. 5 (1994) 482.

[12] T. Girardeau, K. Bouslykhane, J. Mimault, J.P. Villain, P. Chartier, Thin Solid Films 283 (1996) 67.

[13] A. Fontaine, P. Lagarde, A. Naudon, D. Raoux, D. Spanjaard, Phila. Mag. B 40 (1979) 17.

[14] J.J. Rehr, R.C. Alberts, Rev. Mod. Phys. 72 (2000) 621.

[15] P.A. Lee, J.B. Pendry, Phys. Rev., B 11 (1975) 2795.

[16] A.G. McKale, G.S. Knapp, S.K. Chan, Phys. Rev., B 33 (1986) 841.

[17] I. Petrov, F. Adibi, J.E. Greene, L. Hultman, J.-E. Sundgren, Appl. Phys. Lett. 63 (1) (1993) 36. 